The Institutional Repository IRUA is a subset of the Academic bibliography. As of 1991 the UA library produces an academic bibliography for the University of Antwerp (UA). IRUA contains scientific publications of UA researchers. If allowed the fulltext of the publications are made available.

This paper was published in 'Applied optics' and is made available as an electronic reprint with the permission of OSA. The paper can be found at the following URL on the OSAwebsite: http://dx.doi.org/doi:10.1364/AO.48.000941. Systematic or multiple reproduction or distribution to multiple locations via electronic or other means is prohibited and is subject to penalties under law. 


\title{
Tomographic imaging of macroscopic biomedical objects in high resolution and three dimensions using orthogonal-plane fluorescence optical sectioning
}

\author{
Jan A. N. Buytaert* and Joris J. J. Dirckx \\ Laboratory of BioMedical Physics, University of Antwerp, Groenenborgerlaan 171, B-2020 Antwerpen, Belgium \\ *Corresponding author: jan.buytaert@ua.ac.be
}

Received 18 August 2008; revised 15 December 2008; accepted 19 December 2008; posted 8 January 2009 (Doc. ID 99982); published 3 February 2009

\begin{abstract}
A new optical-fluorescence microscopy technique, called HR-OPFOS, is discussed and situated among similar OPFOS-implementations. OPFOS stands for orthogonal-plane fluorescence optical sectioning and thus is categorized as a laser light sheet based fluorescence microscopy method. HR-OPFOS is used to make tomographic recordings of macroscopic biomedical specimens in high resolution. It delivers cross sections through the object under study with semi-histological detail, which can be used to create threedimensional computer models for finite-element modeling or anatomical studies. The general innovation of this class of microscopy setup consists of the separation of the illumination and observation axes, but now in our setup combined with focal line scanning to improve sectioning resolution. HR-OPFOS is demonstrated on gerbil hearing organs and on mouse and bird brains. The necessary specimen preparation is discussed. (C) 2009 Optical Society of America
\end{abstract}

OCIS codes: $110.2945,110.6880,110.6955,120.4570,170.2520,170.3880$.

\section{Introduction and Background}

Techniques allowing one to study and visualize intact biomedical objects in high resolution and in three dimensions (3-D) have become very popular in the past 2 decades. In particular, x-ray micro computer tomography $(\mu \mathrm{CT})$ to record shadow transmission images [1,2] and magnetic resonance imaging (MRI) to obtain sections of water content $[3,4]$ - soft tissue-are common and technologically far advanced. Aside from the usefulness and popularity of these techniques, the attainable resolution with them is less than $10 \mu \mathrm{m}$ for objects of macroscopic scale. Only recent advances in $\mu \mathrm{CT}$ allow resolution up to $1 \mu \mathrm{m}$ on small objects [2].

Using histological sectioning, submicrometer resolution for cross-sectional images of macroscopic biomedical objects is obtained with light microscopy. Obtaining these slices is, however, work intensive and requires physical (one-time and one-directional)

0003-6935/09/050941-08\$15.00/0

(C) 2009 Optical Society of America slicing and thus destruction of the specimen, and registering and image processing of the sections is difficult [5].

Advanced microscopy techniques such as confocal and two-photon microscopy allow one to obtain highresolution views of certain specimen types, however because of a limited penetration depth of less than $1 \mathrm{~mm}$ this cannot be applied on large and nontransparent objects [6]. Point-by-point scanning in 3-D inevitably results in a long recording time and inherent bleaching of the specimen.

From 1993 up until now, another technique has gained much interest and is being developed frantically: orthogonal-plane fluorescence optical sectioning (OPFOS) microscopy or tomography [7-13]. As this technique delivers microscopic and even histological information, and images soft tissue and bony structures simultaneously and in nearly real time, it fills a void between MRI and $\mu \mathrm{CT}$. The OPFOS method is based on two techniques that are about 100 years old and was introduced by Voie [7].

In the OPFOS method, parallel optical sections through a macroscopic biomedical specimen are 
created by means of a thin laser light plane or sheet, and the fluorescence within the sheet is recorded orthogonally. From such a recorded sequential data stack of parallel OPFOS recordings, 3-D models can be created using 3-D software [10-13]. In most microscopy techniques, the same optical path (and components) is used for the illumination and observation light. In the case of OPFOS, the illumination and viewing axes are separated, an idea first introduced by Nobel Prize winner Richard Zsigmondy [14]. He named his setup ultramicroscopy in 1903 -a name recently reused for the OPFOS method as a tribute to Zsigmondy [15] — and used the technique not for tissue microscopy but to study particles within colloidal solutions. Recently researchers applied this initial use of Zsigmondy's technique again in oceanography of aquatic microbes in their natural setting and renamed this approach thin light sheet microscopy $[16,17]$, and it was also used for tracking and identification of single particles [18]. Voie developed the technique to make actual cross sections. These OPFOS sections are created using light, making the method a noncontacting and nondestructive technique. Thus, specimens can be measured more than once, and sliced in any orientation. Moreover, the separation of illumination and imaging axes in OPFOS delivers the advantage that bleaching of sample regions that are not being imaged is avoided.

The other "ancient" procedure used in OPFOS, is the Spalteholz technique from 1911 [19]. For the laser light plane to be able to pass unscattered and without refraction through the macroscopic object, the biomedical specimen needs to be translucent and have the same refraction index throughout its entire volume. This way, when the specimen is submerged in a fluid of the same refraction index, it appears completely transparent. This refractionindex matching is essential for the OPFOS technique to succeed in creating a penetration depth of centimeters, otherwise it would be limited as in confocal microscopy. After this clearing procedure, specimens can be stained with the fluorescent dye Rhodamine B for extra contrast [7-12]. Sometimes green fluorescent protein (GFP)-labeled transgenic animals are used $[15,20]$, or specimens are measured using just their natural autofluorescence [15]. Several researchers also want to image bony tissue together with soft tissue. As calcium-the main component of bone-scatters light strongly it has to be removed. This is achieved in a decalcification process before the Spalteholz procedure is applied [7,10].

After the introduction of OPFOS, similar or improved designs and setups were issued under many different names, all using a spatial arrangement of illumination and detection like Voie and Zsigmondy. First confocal theta fluorescence microscopy focused on the axial resolution and the point-spread functions of the illumination and observation [21]. Later, the same group introduced a newer version that they called selective plane illumination microscopy (SPIM), which was capable of imaging small but living specimens [20]. The Spalteholz technique could thus not be used anymore, and therefore the specimen needed to possess its own natural transparency as Drosophila larvae or Zebrafish or Medaka embryos do, and they are embedded in agarose. This technique is suited to visualize organogenesis in 3-D. Furthermore, this group also improved the image quality using multiview reconstruction by measuring multiple 3-D datasets of the same object, combined in postprocessing $[20,22]$. With multidirectional SPIM, or mSPIM, they added optical components to create identical light sheet sectioning from opposite sides [23], also adopted by the "new" ultramicroscopy [15]. A typical drawback of the OPFOS techniques is the inherent shadow attenuation: when laser light passes through a sample and encounters a particle or region of less transparency, the light behind it is attenuated, creating the typically shadow striped OPFOS images. In the case of two-sided illumination and sectioning with laser sheets, this problem is countered and image quality is improved. The latest version of the (m)SPIM device has been called digital scanned laser light sheet microscope, or generally called laser light sheet based fluorescence microscopy. However, in fact, all these implementations and names come down to OPFOS.

OPFOS and ultramicroscopy have the capability to image cross sections through macroscopic objects of ten(s) of millimeters in dimension. Lateral resolution in all OPFOS techniques is determined by only the NA of the observation lens and the spatial resolution of the recording device. Axial resolution however is determined by the thickness of the laser light sheet. These sheets are created by focusing a broad laser bundle with a cylindrical lens to a hyperbola, which in a certain zone can be approximated to a plane [10]. The region in which this approximation is valid is called the confocal parameter or depth of field $b$. The sectioning thickness is thus not (everywhere) equal to the minimal $1 / e^{2}$ beam width $d_{0}$ but broadens with the hyperbola with a factor $\sqrt{2}$ near the edges of a recording with width $b$. The actual axial resolutions in OPFOS and ultramicroscopy are thus respectively $20(\sqrt{2} \times 14)$ and $35(\sqrt{2} \times 25) \mu \mathrm{m}$ near the edges of cross sections through large objects $[\underline{8}, 15]$. One can make the minimal beam waist $d_{0}$, or focus of the sectioning hyperbola, smaller by using a larger cylindrical lens aperture, thus making the axial resolution better but the depth of focus $b$ much smaller, limiting the method to small objects of $1 \mathrm{~mm}$ or less. This is done in thin light sheet microscopy, (m)SPIM, DSLM, and also ultramicroscopy, achieving about $8.5(\sqrt{2} \times 6) \mu \mathrm{m}$ axial resolution [15].

For objects the size of tens of millimeters, a new OPFOS design was needed to achieve axial resolutions better than $20 \mu \mathrm{m}$. Such a device is for instance useful in our research on the mammal middle and inner ear, and in other biomedical applications where high-resolution tomographic data is needed in large specimens. We therefore developed the high-resolution OPFOS setup [10]. In Section 2, we 
explain the working principle behind HR-OPFOS, show applications and examples, and give suggestions for future ameliorations.

\section{HR-OPFOS Setup Design}

\section{A. Theoretical Description}

We choose our coordinate system such that the camera records cross-sectional images in the $X-Y$ plane, and parallel slices are sequentially taken along the $Z$ axis. The laser light is projected along the $X$ axis. As briefly mentioned in Section 1, the laser light sectioning plane is created by focusing a broad laser bundle with a cylindrical lens. This type of lens focusses the light in only one dimension-here the $Z$ direction-to a hyperbola. In the waist of the hyperbola, the intensity profile forms the well-known slit diffraction pattern if the lens is homogeneously illuminated:

$$
I(z)=I_{0} \cdot \operatorname{sinc}^{2}\left(\frac{z \pi D_{\text {lens }}}{\lambda f}\right)=I_{0} \cdot\left(\frac{\sin \left(\frac{z \pi D_{\text {lens }}}{\lambda f}\right)}{\frac{z \pi D_{\text {lens }}}{\lambda f}}\right)^{2},
$$

with $D_{\text {lens }}$ the diaphragm of the cylindrical lens, $f$ its focal length, and $\lambda$ the wavelength of the laser light. The origin of $X$ is chosen in the minimal focus. The beam width $d(x)$ of this pattern is often defined at height $I_{0} / e^{2}$ of the sinc profile, as we will do throughout this paper. The minimal beam width $d_{0}$ is twice the beam waist $w_{0}$, which for a homogeneously illuminated aperture of a cylindrical lens comes down to

$$
\begin{gathered}
w_{0}=1.4 f \lambda / D_{\text {lens }}, \\
d_{0}=2 w_{0}, \\
d(x)=2 w(x)=2 w_{0} \sqrt{1+\left(\frac{x}{x_{R}}\right)^{2}}, \\
x_{R}=\frac{\pi w_{0}^{2}}{\lambda} .
\end{gathered}
$$

$x_{R}$ stands for the Rayleigh range and is defined as the location along $X$ from the center where the beam waist has grown with the square root of 2 . The depth of field or the confocal parameter $b=2 x_{R}$ is an important variable as it defines the distance along the $X$ axis in which the hyperbola can be approximated as a plane. This is illustrated in Fig. 1. In a medium with refractive index $n$, the confocal parameter becomes

$$
b_{n}=2 n x_{R}=n b .
$$

Objects have to fit within this region in order to record cross sections with a slicing thickness ranging from $d_{0}$ to $d\left(x_{R}\right)=\sqrt{2} d_{0}$. Notice from Eq. (5) that when we make the beam width $d_{0}$ smaller, the confocal parameter $b$ becomes consequently much smaller, forcing the objects which can be measured

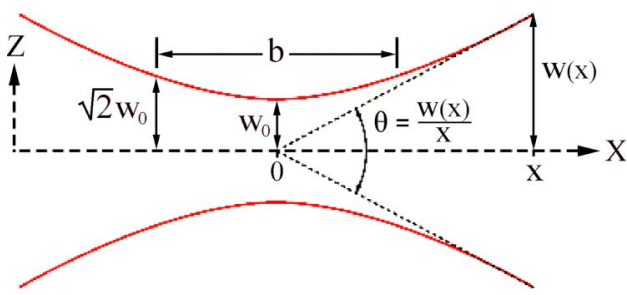

Fig. 1. (Color online) Schematic drawing of the hyperbolic focus of a cylindrical lens. $w_{0}$ is the (minimal) beam waist, $w(z)$ is the half beam width at $z$, and $b$ is the confocal parameter.

to be smaller as well. Up until now, all OPFOS variants had this compromise between slicing resolution and object size.

With high-resolution OPFOS we avoid this concession, thus achieving higher resolution overall. We use a large lens aperture, thus focusing the hyperbola strongly to a very small width $d_{0}$ and consequently small depth of field $b$, but combined with line scanning: Instead of recording real-time 2-D crosssectional images, we record only a 1-D line in this minimal focus along the $Y$ axis and build in nearly real time a cross section by scanning the focal line through the object along the $X$ axis (and along $Z$ for the next sequential slice). This process is illustrated in Fig. $\underline{2}$.

\section{B. Practical Description}

A schematic layout of the setup is shown in Fig. 3 . The laser light progresses along the $X$ axis and is broadened by a Galilean beam expander. The light originates from either a green $532 \mathrm{~nm}$ neodymium laser (DPGL-2050, Photop Suwtech Inc.) or a blue $473 \mathrm{~nm}$ laser (DPSS MPL100 mW, CNI Laser Ltd.). Both beams enter the setup either by passing through or being reflected by a dichroic mirror, so the wavelength can easily be switched. The broad Gaussian parallel beam passes through a field stop, eliminating unnecessary light in the $Y$ direction that might scatter in the setup, and falls onto a cylindrical lens. A double field stop regulates the amount of light along Yaxis and the slit aperture for this lens, creating homogeneous illumination. The aperture and thus beam waist can be set to a desired value. Commercially available cylindrical lenses are singlets. In such lenses, aberration is too large to obtain the very narrow focus needed in HR-OPFOS. Therefore, a custom-made $30 \mathrm{~mm} \times$ $30 \mathrm{~mm}$ achromatic doublet with focal length $80 \mathrm{~mm}$ was manufactured (Moonlight Optics Ltd.). The light is hyperbolically focused in the $Z$ direction to a focal line along the $Y$ axis. The observation direction of the CCD camera (FO442BIC FireWire, FOculus NET $\mathrm{GmbH}$ ) is normal to the $X-Y$ plane. The CCD has $1280 \times 960$ pixels. If we use the setup in OPFOS mode, a 2-D cross-sectional image with this spatial resolution is recorded. In HR-OPFOS mode, however, the camera only records one (or a predetermined number of) 1-D pixel line(s) of 1280 pixels high, exactly in the minimal hyperbola beam waist. The object is moved and line scanned along $X$ to 

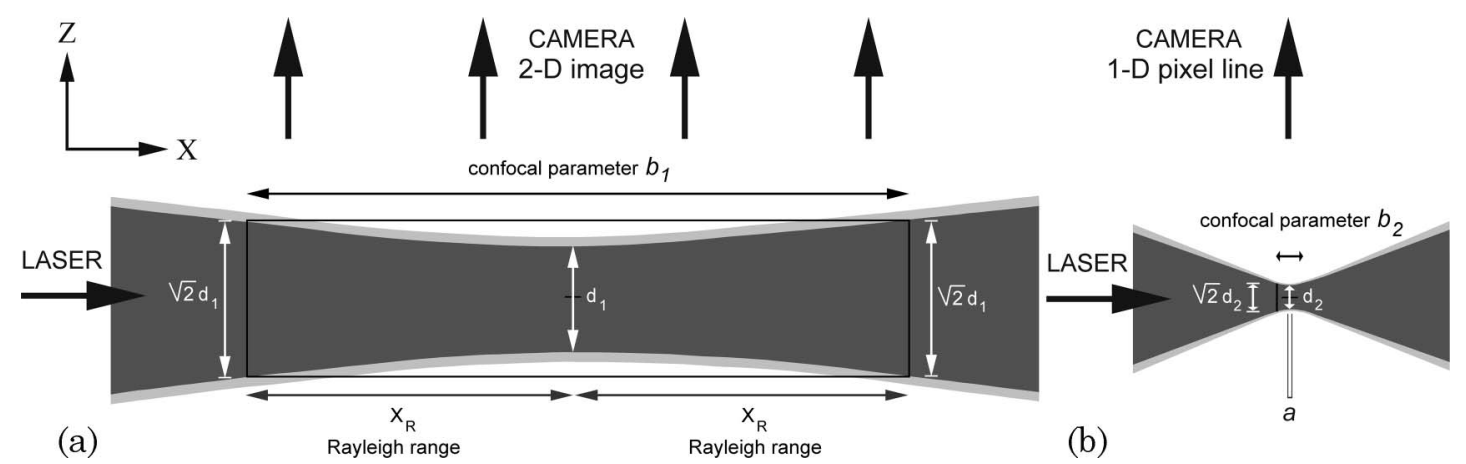

(b)

Fig. 2. (a) OPFOS: the hyperbolic focus is approximated to a planar sheet in the confocal parameter range $b$, with $1 / e^{2}$ thickness $d_{1}$ in the center and $\sqrt{2} d_{1}$ near the edges of the sheet. A 2 -D image is taken orthogonally in the $X-Y$ plane. (b) HR-OPFOS: a larger lens aperture can be used to create a minimal focal line thickness $d_{2}$, as no 2-D sheet is needed. Line scanning of the object along $X$ is performed to obtain a 2 -D cross section.

obtain a 2-D image of "unlimited length" by two orthogonally positioned M112 high-resolution microtranslation stages with C862 Mercury II DC-motor controllers (Physik Instrumente $\mathrm{GmbH}$ ) with a spatial accuracy of $7 \mathrm{~nm}$.

As the CCD camera needs to view and record the light coming from within a certain cross section, the object needs to be completely transparent and fluorescent. Because of the orthogonally separated illumination-observation design in OPFOS, no out-of-focus fluorescence is observed (as opposed to confocal microscopy): the camera is focused on the $X-Y$ laser sheet, and observed light can only originate from within the light sheet, which strongly reduces bleaching. When scanning along the $X$ axis for a section recording (HR-OPFOS), this advantage is no

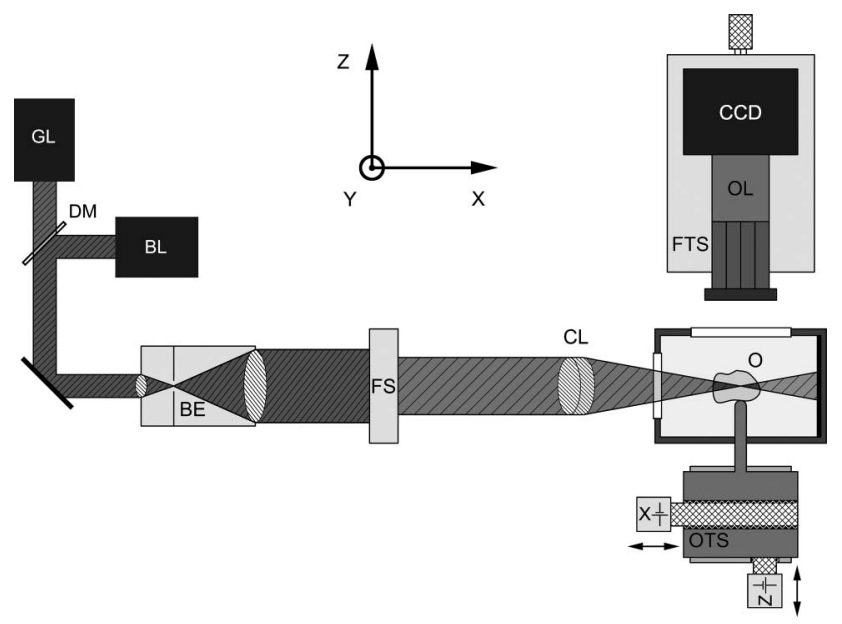

Fig. 3. Schematic drawing of the (HR-) OPFOS instrument. Using a dichroic mirror (DM), blue laser (BL) or green laser (GL) light is broadened by a beam expander (BE). A double field stop (FS) limits the amount of light along $Y$ and determines the slit aperture of the cylindrical lens (CL). The CL focuses the light hyperbolically through a window in the specimen container into the object $(\mathrm{O})$ under study. At the back of the container a black wall is placed, absorbing all laser light. A second window in the specimen chamber, filled with fluid, allows the CCD with objective lens (OL) to record a cross section. The focus translation stage (FTS) has to be calibrated only once. The object is scanned along $X$ and at different $Z$ depths by a two-axis motorized translation stage (OTS). longer fully valid, however the occurrence of bleaching is still far less than with confocal microscopy [24]. To maintain the focus while slicing the object a different $Z$ depths, the same amount of refraction index has to be kept between the laser sheet and the CCD. Some authors move the laser plane through the object by moving the laser itself or more ingeniously using mirrors [23], which requires that the observation lens and camera be refocused continually. Other authors moved the specimen chamber in which the specimen was fixed [9]. The laser part of the setup does not need to move, however the imaging part does need to refocus as a different amount of refractive material is between the sheet and the CDD. Finally, there are authors who rotated the object, an acceptable choice only if the object has axial symmetry like a cochlea [8]. In this case, the camera focus is automatically maintained. In our instrument, we also maintain a fixed focus by moving the object (which has the same refractive index as the fluid it is in) in the specimen chamber, while keeping the laser and observation components of the setup itself stationary. This way the optical path length remains the same and the setup remains aligned, which delivers a better stability of the setup to obtain micrometer resolution. It is important to make sure that the camera detector is perfectly parallel to the $X-Y$ plane, that its pixel columns are exactly parallel to the $Y$ axis, and that the cylindrical lens is parallel to the $Y-Z$ plane. The specimen container is machined from aluminum and has windows made from optical quality glass and sealed with O-rings (Viton, DuPont), which are Spalteholz fluid resistant, allowing the laser to enter the chamber. The chamber is filled with Spalteholz clearing solution, and its back side is blackened to absorb the transmitted laser light. Another window is present in the container for observational purposes for the camera. The camera itself is positioned on a translation stage and has observation lenses with different magnification objectives $(2 \times, 5 \times$, and $10 \times \mathrm{M}$ Plan APOs with $\mathrm{NA}=0.056, \mathrm{NA}=0.14$, and $\mathrm{NA}=0.28$, respectively, Mitutoyo Corp.). Combined with these microscope objectives we apply a high-pass color filter, removing 
the remaining scattered laser light from the images and transmitting only fluorescence light to the CCD. Once calibrated, this setup needs no physical interaction or refocusing.

The (HR-)OPFOS instrument is fully automated by custom-made operating software written in the programming language Matlab R2008 (Mathworks Inc.). It allows total digital control over the setup, which comprises recording images, timing and performing displacement of all motors, saving of the data, and optional image processing.

\section{Specimen Preparation}

OPFOS requires the biomedical specimen to undergo an elaborate specimen preparation to become transparent and fluorescent. Suppose the specimen consists of both soft tissue and bony structures, e.g., a gerbil middle ear containing muscle, blood vessels, tendon, and bone.

\section{A. Dissection}

The bulla containing the middle and inner ear is harvested from the skull of an euthanized gerbil. All blood has to be removed from the specimen as coagulate blood will not become optically transparent. This is achieved by premortem injection of 100 units / $200 \mathrm{~g}$ heparin (LEO Pharma) and postmortem perfusion with buffered saline. The specimen is then fixated in a $10 \%$ formalin solution, neutrally buffered.

\section{B. Decalcification}

The primary tissue of bone-osseous tissuemineralized connective tissue, consisting of a collagen matrix and calcium phosphate. Because of the calcium, bone is nontransparent for laser light and scatters it around. Removal of the calcium from an OPFOS specimen is thus also essential.

Decalcification is achieved by placing the specimen in a $10 \%$ demineralized water solution of dihydrate ethylene-diamine-tetra acetic acid disodium salt (EDTA, CAS No. 6381-92-6, VWR International). The calcium is chelated by the EDTA, which allows it to be transported outside of the specimen more easily. The complete $\mathrm{Ca}^{++}$removal process takes a very long time, many weeks or months even, and needs the solution to be refreshed daily. Decalcification is however much accelarated by exposing the specimen to low-power microwave radiation [25]. One week in our in-house built microwave setup with cooling and daily refreshing of the EDTA solution suffices. Afterwards, rinsing with demineralized water is necessary, as (chelated) EDTA still scatters laser light (and is x-ray opaque).

\section{Refractive-Index Matching}

Clearing of the specimen to allow laser light sheets to virtually section the object is the third major step. When the refraction index of all tissue in a specimen (without blood or calcium) is made equal and it is submerged in fluid of the same index, it becomes completely transparent: no diffusion or refraction of light is present.

To achieve this, all water has to be extracted from the sample and replaced by Spalteholz fluid [19]. This fluid consists of 5 parts methyl salicylate (CAS No. 119-36-8, Merck) and 3 parts benzyl benzoate (CAS No. 120-51-4, Prolabo), and is not soluble in water. Therefore dehydration is first required using a gradually climbing ethanol series $(20,40,60,80,100$, $100 \%$ each $24 \mathrm{~h}$ ), followed by a similar gradually climbing Spalteholz series in ethanol. We measured the refraction index of Spalteholz at $n=1.53876$. The result is a cleared specimen with this refraction index as well.

\section{Fluorescence}

Finally, it might be useful to stain the specimen with (possibly several different) fluorescent dyes. Bathing the sample in Spalteholz solution with $5 \times$ $10^{-4} \mathrm{mg} / \mathrm{ml}$ tetramethyl rhodamine B isothiocynate (TRITC, CAS No. 95197-95-8, Fluka) is useful to improve contrast in combination with a green laser.

Recently we started using a blue laser, allowing us to record cross sections using autofluorescence of specimens without staining. We suspect collagen and elastin to be responsible for the autofluorescent light.

\section{Applications}

\section{A. Resolution}

The in-plane resolving power depends on the NA of the microscope objective used for observation. Hence, lateral resolution ranges from $1 \mu \mathrm{m}$ for the $10 \times, 2 \mu \mathrm{m}$ for the $5 \times$, to $5 \mu \mathrm{m}$ for the $2 \times$ lens, which we verified with a resolution target (U.S. Air Force 1951).

The CCD camera can take a 2-D image of $1280 \times$ 960 pixels when used in OPFOS mode of the setup. In HR-OPFOS, it records one (or several) 1-D pixel column(s) of 1280 pixels in the center of the hyperbolic focus. With the $2 \times$ lens, this corresponds to an object height of $3755 \mathrm{~mm}$, which is (with our camera) the limiting size of the objects in the $Y$ dimension. In the $X$ direction, no such limit exists as line scanning is applied there. So objects can easily be $20 \mathrm{~mm}$ long. Along the $Z$ axis, there is also no immediate limit to the number of slices, except for the quality of transparency deeper inside the specimen. In a perfectly cleared specimen, the imaging depth is still limited by the working distance of the objective. As in our system we are using objectives with a working distance of several centimeters, no practical limitation for our applications has been found.

The slicing or axial resolution depends on the thickness of the sectioning laser sheet. In OPFOS mode, when using the full aperture of $30 \mathrm{~mm}$ - the dimensions of the cylindrical lens-with homogeneous illumination of $532 \mathrm{~nm}$, a focal thickness of $d_{1}=2.0 \mu \mathrm{m}$ is obtained in Spalteholz fluid, but a confocal parameter $b_{1 n}$ of only $18 \mu \mathrm{m}$. This is not good 
enough for macroscopic objects of, e.g., dimension $16 \mathrm{~mm}$. To image such an object, a lens aperture of $1 \mathrm{~mm}$ has to be used, because this delivers $d_{1}=$ $40 \mu \mathrm{m}$ and $b_{1 n}=16.1 \mathrm{~mm}$. Near the edges of the 2 -D recording of the cross section, a slicing thickness of $\sqrt{2} d_{1}=56 \mu \mathrm{m}$ is obtained. An aperture of $1 \mathrm{~mm}$ will also block the major part of the laser light, losing much of the intensity.

In HR-OPFOS on the other hand we can use the full aperture of $30 \mathrm{~mm}$ with beam width $d_{2}=$ $2.0 \mu \mathrm{m}$. There is no need to obtain a larger confocal parameter here or pay attention to the hyperbolic profile, as we record only in the line of focus.

Measurements with HR-OPFOS of the pointspread function of submicrometer fluorescent beads (Polysciences Inc.) showed a slicing resolution of $2 \mu \mathrm{m}$ [10]. Another verification was done, recording cross sections through biomedical specimens less than $2 \mu \mathrm{m}$ apart. When these were subtracted from each other, new resolved information content in every section was clearly noticeable.

\section{B. Ear Measurements}

In hearing research, the gerbil is one of the most common animal models. In Fig. 4(a) we show a cross section, built from 1700 individually scanned lines along $X$ in $1.5 \mu \mathrm{m}$ steps, of a cochlea in a left gerbil inner ear. This HR-OPFOS recording shows histological detail, such as small blood vessels and, for instance, the very thin Reissner's membrane. A movie (Media 1) containing more cross sections at depths increasing with $\Delta z=15 \mu \mathrm{m}$ is included with Fig. 4(a). Figure 4(b) shows a section built from $\Delta x=0.7 \overline{5 \mu \mathrm{m}}$ steps through the stapes in the middle ear. Notice how internal bone structure can be studied and how the incudostapedial joint can be clearly observed. These recordings are made in stained samples with the TRITC dye.

When recording many sequential cross sections along $Z$, a $3-\mathrm{D}$ data stack is obtained with high and nearly isometric resolution. This was impossible with OPFOS before because of the thick slicing in macroscopic objects. To prove that we achieve isometric resolution, a data stack of $X-Y$ HR-OPFOS sections is resectioned to orthogonal planes, showing comparable image quality in Fig. 5 .

Using specialized software such as Modeller, Mesh3D, or Amira, 3-D computer models can be created. From the data in Fig. $\underline{5}$, we obtain a 3-D representation of the bony stapes with surrounding soft tissue in Fig. 6(a). This HR-OPFOS surface or volume model has many faces or voxels. The right image of Fig. 6(a) shows a decimated version, still of good quality. A movie of the 3-D stapes mode (Media 3 ) is available online with this paper. This procedure can of course be executed for an entire middle ear ossicle chain, resulting in the model shown in Fig. 6, which can be used to simulate the complex transfer function of acoustical energy from a sound wave in air to a pressure wave in fluid.

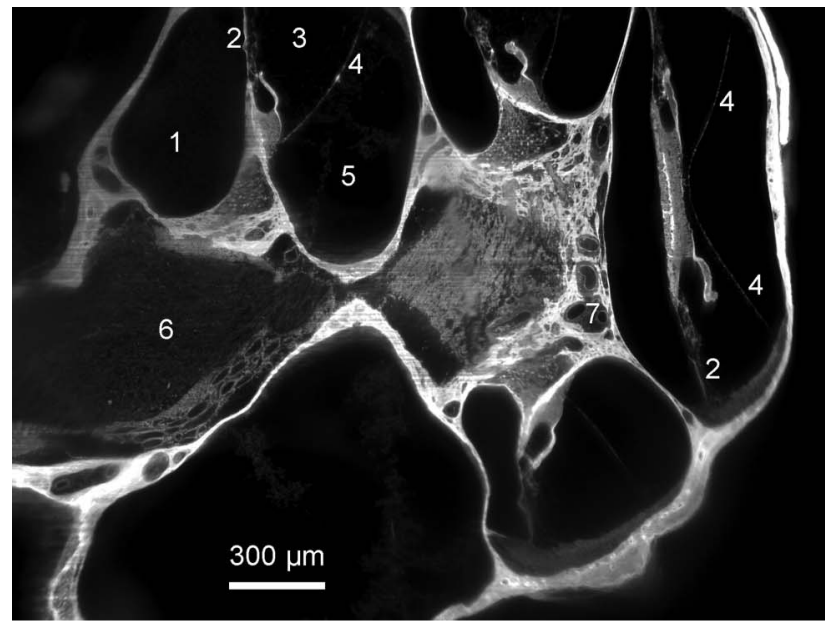

(a)

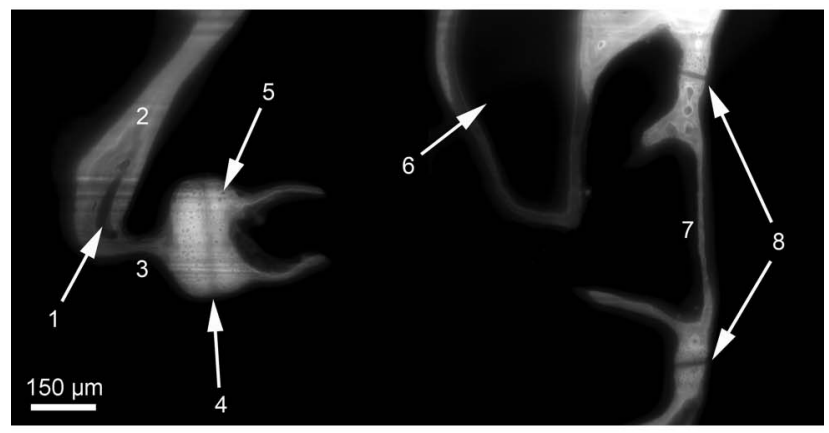

(b)

Fig. 4. (a) Cross section of a right gerbil cochlea obtained (every $30 \mu \mathrm{m}$ in Media 1) with HR-OPFOS of $1.92 \mathrm{~mm} \times 2.55 \mathrm{~mm}$ $(1280 \times 1700$ pixels $)$. Every image is scanned in 1700 steps of $1.5 \mu \mathrm{m}$, with lateral and axial resolution of $2 \mu \mathrm{m}$. 1, scala tympani; 2 , basilar membrane; 3 , scala media; 4 , Reissner's membrane; 5 , scala vestibuli; 6 , modiolus; 7 , blood vessels. See for more cross sections. (b) Cross section of a right gerbil stapes obtained with HROPFOS of $0.96 \mathrm{~mm} \times 1.88 \mathrm{~mm}(1280 \times 2500$ pixels $)$ with lateral resolution of $1 \mu \mathrm{m}$ and axial resolution of $2 \mu \mathrm{m}$. 1, duct within incus; 2 , incus; 3 , lenticular process; 4 , articulation; 5 , calcified cartilage within stapes head; 6 , stapedial artery; 7 , stapes footplate; 8 , annular ligament footplate.

\section{Brain Measurements}

It is also possible to image cross sections in cerebellum tissue with (HR-)OPFOS. In Fig. 7 (Media 4) such recordings are shown from a mouse brain, in OPFOS mode. The autofluorescence of the brain tissue is used here. Much detail can be distinguished, such as axon bundles, different brain regions, and small blood vessels. Note that these images are obtained in an adult mouse brain of about $15 \mathrm{~mm}$ diameter, not in small brains of mouse embryos.

Apart from remaining blood, water, or calcium, there is one more thing that can be a major problem in (HR-)OPFOS imaging: myelin. This is an electrically insulating dielectric phospholipid layer that surrounds only the axons of many neurons. Myelin is not optically transparent and scatters laser light 


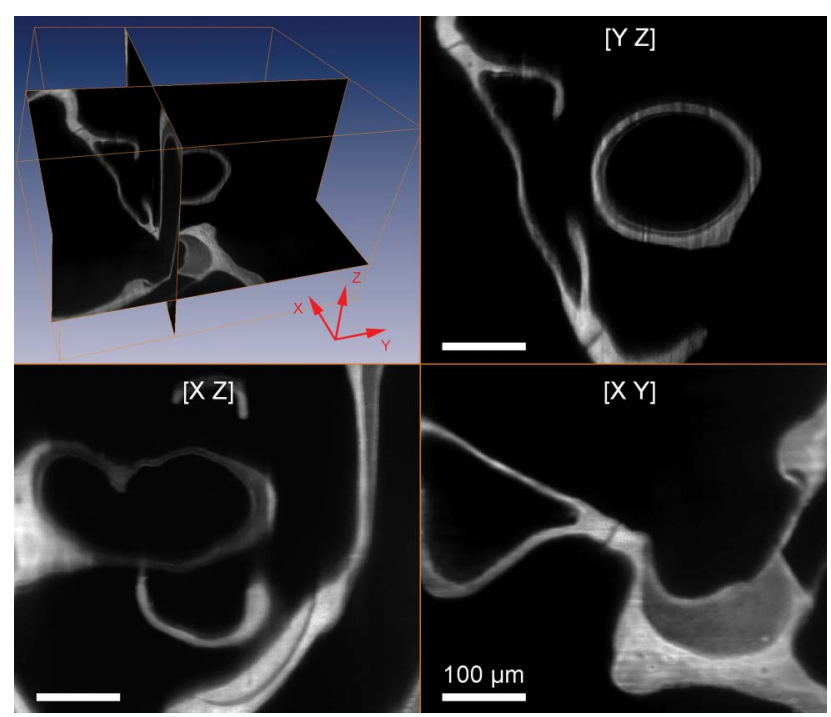

Fig. 5. (Color online) After recording 435 scanned cross sections of $1280 \times 1280$ pixels with HR-OPFOS in the $X-Y$ plane, $2 \mu \mathrm{m}$ apart, computer software can resection this 3-D data stack to $X-Z$ or $Y-Z$ with the same image quality. See Media 2 for more cross sections in $X-Y$. In Media 2 an entire stack of 385 HR-OPFOS cross sections $(1.92 \mathrm{~mm} \times 2.55 \mathrm{~mm})$ each $2.5 \mu \mathrm{m}$ apart is shown with 13 sections/s, which amounts to a depth progress of $32.5 \mu \mathrm{m} / \mathrm{s}$. Readers familiar with the anatomy may see the stapedial muscle attaching to the stapes head, and the stapedial artery running through the crurae.

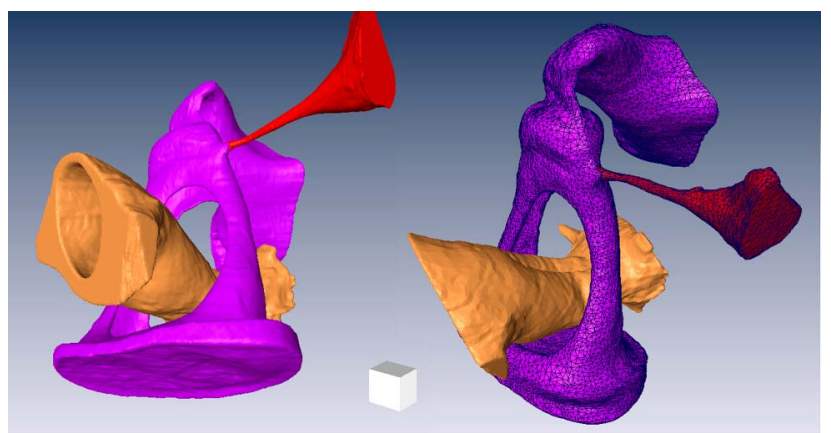

(a)

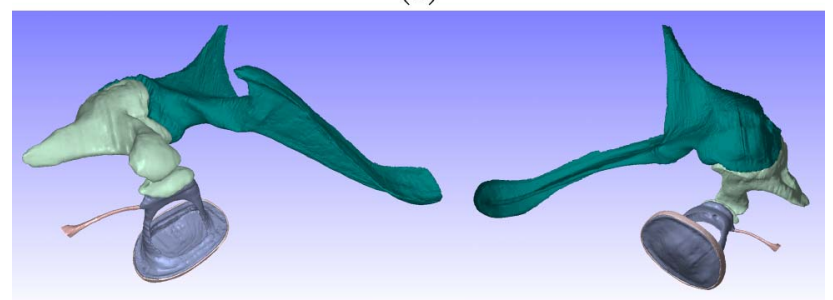

(b)

Fig. 6. (Color online) (a) From a 3-D (HR-) OPFOS data stack we can create $3-\mathrm{D}$ reconstructions. The model shown here originates from the data stack in Fig. 5, showing a part of the incus attaching to the stapes, the stapedial artery running through the stapes "legs" and the stapedial muscle connecting at the stapes head (voxels $1.5 \mu \mathrm{m} \times 2 \mu \mathrm{m} \times 2 \mu \mathrm{m}$ ). The cube in the image has dimensions $150 \mu \mathrm{m}$. See Media 3 for a 3-D impression of the model. (b) This 3 -D model shows a reconstruction of a complete ossicle chain, comprising bone structures (malleus, incus, and stapes) and soft tissue structures (annular ligament around the stapes and the stapedial muscle) (voxels $4 \mu \mathrm{m} \times 4 \mu \mathrm{m} \times 4 \mu \mathrm{m}$ ).

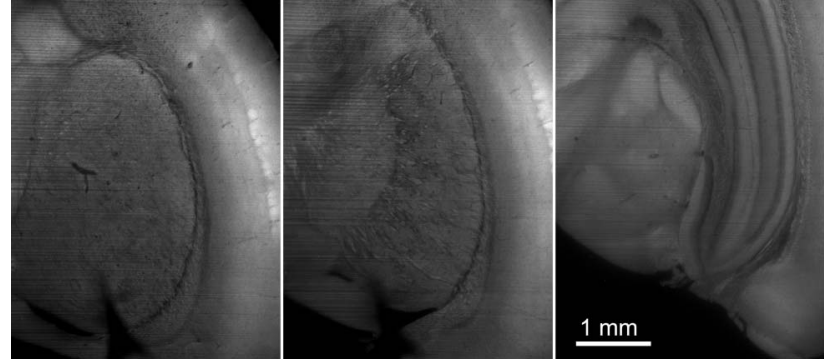

Fig. 7. Three OPFOS cross sections $(4.8 \mathrm{~mm} \times 3.6 \mathrm{~mm})$ at different depths through a mouse brain are shown. The natural autofluorescence of the specimen clearly allows one to distinguish histological detail. See Media 4 for more cross sections in $X-Y$. The movie shows 24 sections/s, which amounts to a depth progress of $240 \mu \mathrm{m} / \mathrm{s}$.

around, creating blurry images or regions. This might be a problem when imaging biomedical samples containing nerve fibers, especially brains. Myelin is much less present in younger animals and embryos. As myelin is a lipid, some type of defattening approach might solve the opacity problem in specimens containing many nerve fibers.

\section{Conclusion}

The OPFOS technique, in all its variants, is a valuable technique in many fields, from detection of single particles to 3-D imaging of macroscopic biomedical structures. It images soft and even bony tissue at the same time and its orthogonal separation of illumination and viewing axes offers many advantages: no out-of-focus illumination, better axial resolution, less bleaching, (nearly) real-time sectioning, no registration difficulties, etc. Specimen preparation is quite elaborate and sensitive to artifacts.

We presented here a high-resolution OPFOS instrument design, achieving high microscopic resolution on macroscopic objects of tens of millimeters in size. There are some ameliorations still to be implemented, improving HR-OPFOS even further, such as: two-sided illumination; functional staining with several dyes; inserting a shutter that allows only laser light onto the object when the camera is running, thus reducing bleaching even more; and using a camera with many more pixels in the $Y$ direction so we can measure even "higher" objects (along $X$ and $Z$ we can scan). Other authors use ingenious image (post) processing and the combination of several datasets, which can easily be added to and implemented with our technique as well to attribute to even better quality cross sections.

J. A. N. Buytaert has a fellowship of the Research Foundation-Flanders (FWO-Vlaanderen) and received financial support from the Rosa Blanckaert Foundation. We thank F. Wiese for his technical support and J. Van Hecke for his assistance with the multimedia files. 


\section{References}

1. S. L. R. Gea, W. F. Decraemer, and J. J. J. Dirckx, "Region of interest micro-CT of the middle ear: A practical approach," J. X-Ray Sci. Technol. 13, 137-147 (2005).

2. B. Masschaele, V. Cnudde, M. Dierick, P. Jacobs, L. V. Hoorebeke, and J. Vlassenbroeck, "UGCT: new x-ray radiography and tomography facility," Nucl. Instrum. Meth. A 580, 266-269 (2007).

3. M. M. Henson, O. W. Henson, S. L. Gewalt, J. L. Wilson, and G. A. Johnson, "Imaging the cochlea by magnetic resonance microscopy," Hear. Res. 75, 75-80 (1994).

4. J. M. Tyszka, S. E. Fraser, and R. E. Jacobs, "Magnetic resonance microscopy: recent advantages and applications," Curr. Opin. Biotechnol. 16, 93-99 (2005).

5. W. J. Weninger, S. Meng, J. Streicher, and G. B. Müller, "A new episcopic method for rapid 3-D reconstruction: applications in anatomy and embryology," Anat. Embryol. 197, 341-348(1998).

6. F. Helmchen and W. Denk, "Deep tissue two-photon microscopy," Nat. Methods 2, 932-940 (2005).

7. A. Voie, D. Burns, and F. Spelman, "Orthogonal-plane fluorescence optical sectioning: three-dimensional imaging of macroscopic biological specimens," J. Microsc. 170, 229236 (1993).

8. A. Voie, "Imaging the intact guinea pig tympanic bulla by orthogonal-plane fluorescence optical sectioning microscopy," Hear. Res. 171, 119-128 (2002).

9. W. Valk, H. Wit, J. Segenhout, F. Dijk, J. van der Want, and F. Albers, "Morphology of the endolymphatic sac in the guinea pig after an acute endolymphatic hydrops," Hear. Res. 202, 180-187 (2005).

10. J. A. N. Buytaert and J. J. J. Dirckx, "Design and quantitative resolution measurements of an optical virtual sectioning 3-D imaging technique for biomedical specimens, featuring $2 \mu \mathrm{m}$ slicing resolution," J Biomed. Opt. 12, 014039 (2007).

11. J. A. N. Buytaert and J. J. J. Dirckx, "High-resolution 3-D imaging of middle ear ossicles and soft tissue structures of intact gerbil temporal bones using orthogonal-plane fluorescence optical sectioning," in Middle Ear Mechanics in Research and Otology, A. Eiber and A. Huber, eds. (World Scientific, 2007), pp. 282-288.

12. R. Hofman, J. Segenhout, J. A. N. Buytaert, J. J. J. Dirckx, and H. Wit, "Morphology and function of Bast's valve; Additional insight in its functioning using 3D-reconstruction," Eur. Arch. Oto-Rhino-L. 265, 153-157 (2008).
13. J. A. N. Buytaert and J. J. J. Dirckx, "High-resolution virtual optical-sectioning imaging and tomography for 3-D modeling of biomedical specimens," in Biomedical Optics Topical Meeting, OSA Technical Digest (CD) (Optical Society of America, 2008), paper BWG5.

14. H. Siedentopf and R. Zsigmondy, "Uber die Sichtbarmachung und Grössenbestimmung ultramikroskopischer Teilchen," Ann. Phys. 4, (1903).

15. H. Dodt, U. Leischner, A. Schierloh, N. Jährling, C. Mauch, K. Deininger, J. Deussing, M. Eder, W. Zieglgänsberger, and K. Becker, "Ultramicroscopy: three-dimensional visualization of neuronal networks in the whole mouse brain," Nat. Methods 4, 331-336 (2007).

16. E. Fuchs, J. Jaffe, R. Long, and F. Azam, "Thin laser light sheet microscope for microbial oceanography," Opt. Express 10, 145-154 (2002).

17. C. Davis, "Optical imaging of ocean plankton: a fantastic voyage," in Digital Holography and Three-Dimensional Imaging, OSA Technical Digest (CD) (Optical Society of America, 2008), paper DMB1.

18. J. G. Ritter, R. Veith, J.-P. Siebrasse, and U. Kubitscheck, "High-contrast single-particle tracking by selective focal plane illumination microscopy," Opt. Express 16, 7142-7152 (2008).

19. W. Spalteholz, Uber das Durchsichtigmachen van menschlichen und tierischen Präparaten (Verlag S. Hirzel, 1911).

20. J. Swoger, J. Bene, F. D. Wittbrodt, and E. H. Stelzer, "Optical sectioning deep inside live embryos by selective plane illumination microscopy," Science 305, 1007-1009 (2004).

21. S. Lindek and E. H. Stelzer, "Confocal theta microscopy and 4Pi-confocal theta microscopy," Proc. SPIE 2184, 188-194 (1994).

22. J. Swoger, J. Huisken, and E. H. K. Stelzer, "Multiple imaging axis microscopy improves resolution for thick-sample applications," Opt. Lett. 28, 1654-1656 (2003).

23. J. Huisken and D. Y. R. Stainier, "Even fluorescence excitation by multidirectional selective plane illumination microscopy (mSPIM)," Opt. Lett. 32, 2608-2610 (2007).

24. P. J. Verveer, J. Swoger, F. Pampaloni, K. Greger, M. Marcello, and E. H. Stelzer, "High-resolution three-dimensional imaging of large specimens with light sheet-based microscopy," Nat. Methods 4, 311-313 (2007).

25. S. Tinling, R. Giberson, and R. Kullar, "Microwave exposure increases bone demineralization rate independent of temperature," J. Microsc. 215, 230-235 (2004). 